Vol. 5, No. 2, 2019

\begin{abstract}
Ihor Kuzio ${ }^{1}$, Bogdan Vasyliv ${ }^{2}$, Vitaliy Korendiy ${ }^{3}$, Volodymyr Borovets ${ }^{4}$
1. Department of Mechanics and Automation Engineering, Lviv Polytechnic National University, Ukraine, Lviv,

S. Bandery Street 12, E-mail: Ihor.V.Kuzo@lpnu.ua, ORCID 0000-0001-9271-6505

2. Department of Hydrogen Technologies and Alternative Energy Materials, Karpenko Physico-Mechanical Institute, Ukraine, Lviv, Naukova Street 5, E-mail: mechengin1111@gmail.com, ORCID 0000-0002-8827-0747 3. Department of Mechanics and Automation Engineering, Lviv Polytechnic National University, Ukraine, Lviv,

S. Bandery Street 12, E-mail: vitaliy.nulp@gmail.com, ORCID 0000-0002-6025-3013

4. Department of Mechanics and Automation Engineering, Lviv Polytechnic National University, Ukraine, Lviv,

S. Bandery Street 12, E-mail: volbor1@gmail.com, ORCID 0000-0002-7443-3066
\end{abstract}

\title{
OPTIMIZATION OF GEOMETRIC PARAMETERS OF A SEMI-SPHEROIDAL SOLID OXIDE FUEL CELL ANODE USING THE 3D STRESS AND STRAIN DISTRIBUTION GRAPHS
}

Received: August 20, 2019 / Revised: August 27, 2019 / Accepted: August 30, 2019

(C) Kuzio I., Vasyliv B., Korendiy V., Borovets V., 2019

Abstract. The purpose. Determination of radii ranges for cylindrical and convex (semispheroidal) parts of the solid oxide fuel cell (SOFC) semi-spheroidal shape anode based on stress and strain parameters calculated; comparison of 3D graphs of stress/strain distribution in anodes of proposed and spheroidal shapes; substantiation of the semi-spheroidal anode potential to withstand deformation and stress gradient under operational conditions.

The research method. The object of research is a solid oxide fuel cell anode of a semispheroidal shape loaded with a fixing pressure along the closed-loop fixing and with an external gas pressure applied to the anode working surface. Stress and strain distributions in the anode were calculated by finite element analysis using software for calculating three-dimensional tasks Mechanical Desktop 6 Power Pack. Three-dimensional (3D) dependences of stress/strain distribution in anodes of proposed and spheroidal shapes at a variety of $R / R_{c}$ ratios were plotted. Based on these curves, 3D surfaces of stress distribution along the axis and closed-loop fixing of semi-spheroidal shape anodes were constructed.

Results. Three-dimensional curves of the graphic intersections of the surfaces of stress distribution along the axis and closed-loop fixing of semi-spheroidal shape anodes, with their projections on three coordinate planes, were plotted. The curves display the values of balanced stresses depending on geometric parameters. Domains of these curves were also defined.

The scientific novelty. The proposed method of building 3D surfaces of stress/strain distribution in anodes depending on their geometric parameters shows for the first time that there exists an area of geometric parameters that allows the appropriate stress level to be reached ensuing safe long-term operation of the semi-spheroidal shape anode. The domain of this area was graphically defined. Based on the plotted isolines showing levels of strain in anodes with the $0.5 \mathrm{~mm}, 1 \mathrm{~mm}$, and $1.5 \mathrm{~mm}$ thick cylindrical parts and a variety of spheroid to cylinder radii ratios, an advantage of a semi-spheroidal shape anode over spheroidal one was substantiated.

The practical value. The obtained calculation results and their 3D graphical interpretation can be used in the study of the stress state and, respectively, to evaluate the strength and stiffness of the anode supported SOFCs of various shapes.

Keywords: solid oxide fuel cell, semi-spheroidal and spheroidal shape anodes, finite element analysis, stress and strain distributions, 3D graph, lifetime. 


\section{Introduction}

The advantages of a solid oxide fuel cell (SOFC) such as high efficiency, fuel flexibility, long-term stability, and relatively low cost show the prospect of using this renewable energy source. During operation, it produces electricity directly due to oxidation of a fuel.

The anode supported SOFC is one of the up-to-date design solutions. It consists of the relatively thin electrolyte and cathode layers formed on the surface of a thick anode substrate. As materials of anode substrate, YSZ-Ni and ScCeSZ-Ni cermets are widely used [1] - [5]. Preparation of these substrates involves up-to-date powder metallurgy methods of sintering porous ScCeSZ-NiO or YSZ-NiO (zirconia stabilized with scandia and ceria (or with yttria), i.e. ScCeSZ or YSZ, and $\mathrm{NiO}$ ) ceramic parts of required geometry and sizes, followed by their reduction in a hydrogen-containing gas atmosphere at high temperature (a few hundred degrees Celsius). As a result of $\mathrm{NiO}$ reduction to metallic nickel, a conductive nickel network is formed in a porous ceramic matrix.

\section{Problem Statement}

Since an elementary (single) SOFC is considered as a layered composite consisting of an anode, an electrolyte, and a cathode [1], [6] - [9], there exists a need to optimize the structure of these components, in particular, because of the susceptibility of Ni-containing anode substrates to operating hydrogencontaining gas atmospheres [10] - [13]. The task is to improve the lifetime of a SOFC and to lower the cost of electricity that it produces. For this reason, researchers develop single SOFCs of various shapes aiming at lowering stresses and strains that arise in a fuel cell during operation.

\section{Review of Modern Information Sources on the Subject of the Paper}

It is known that an anode of tubular shape is widely used in the SOFC industry [14]. The main advantage of such an anode is that its operating surface area is of maximum possible value from the geometrical and structural points of view [15]. However, the stresses occurring at sealing the environment supply system make some limitations in the length of a tubular SOFC. The size of the SOFC is additionally limited by the pressure of an operating environment.

There exist other design solutions of a SOFC anode, for example, of truncated cone shape [16]. It is characterized by a high value of the ratio of surface area to volume of the anode which corresponds to a large operating surface area. However, this area is a quarter smaller than that of the anode of tubular shape. The battery of cone-shaped SOFCs is assembled by imposing a wider part of each next cell on a narrower part of a previous cell. However, one of the main disadvantages of such SOFCs is that substantial tensile stress arises in a wider part of each cell during the operation. As a result, its lifetime is reduced due to microcracks initiated at the edge.

Recently, a relatively cheap technology of tape casting of anode plates has been developed which allowed the uniform anode microstructure to be formed. Using this technology, a plane anode of rectangular shape [17], [18], and a round-shaped (cylindrical) anode [19], [20] with flat working surfaces are being manufactured. Thin electrolyte and cathode layers are also formed on the anode substrate using this technology. The thickness of a flat anode is limited because of the requirement for its penetration ability. Since that, the stresses arise when assembling elementary fuel cells in a stack that cause limitations in an operating surface area of such a flat SOFC. Besides, these stresses are not distributed uniformly in a plane anode of rectangular shape [21] whereas a round shape of the cylindrical anode provides the uniform distribution of the axially symmetric stresses arising when assembling elementary fuel cells in a stack. However, the cylindrical anode does not provide the required deformation resistance and, consequently, the required lifetime of a SOFC because of limitations in thickness.

A spheroidal shape of a SOFC anode has been proposed recently [22]. The anode of such a shape provides improved resistance to deformation. We substantiated such a spheroidal shape anode from the point of view of its ability to withstand deformation and stress gradient under operational conditions, especially, the stresses arising along the anode axis [23].

The abovementioned features of the SOFC anode design affect the cost of electricity that a fuel cell produces and its performance per unit volume of the anode. 
Taking into account the requirement on the penetration ability of an anode substrate and, accordingly, its thickness, a semi-spheroidal shape of a SOFC anode can be proposed. It provides improved resistance to deformation as compared to the anode of a cylindrical shape.

\section{Objectives and Problems of Research}

The purpose of this work is to determine radii ranges for cylindrical and convex (semi-spheroidal) parts of the SOFC semi-spheroidal shape anode based on stress and strain parameters calculated, compare 3D graphs of stress/strain distribution in anodes of proposed and spheroidal shapes, and substantiate the semi-spheroidal anode potential to withstand deformation and stress gradient under operational conditions.

\section{Experimental and Calculation Methods}

A solid oxide fuel cell anode can be composed of a mixture of YSZ powders (cubic structured zirconia $\mathrm{ZrO}_{2}$ stabilized with $8 \mathrm{~mol} \% \quad \mathrm{Y}_{2} \mathrm{O}_{3}$ ) with the addition of $20-50 \mathrm{wt} \% \mathrm{NiO}$, since it is the most commonly used powder material for such purpose. The mixture is compressed under a pressure of 16$20 \mathrm{MPa}$ at a temperature of $20{ }^{\circ} \mathrm{C}$ in a cylindrical mold having the concave bottom of the spheroidal shape by a punch having a flat contacting surface. As a result, a cylinder shape pressed body with a flat top surface and a convex bottom one is formed (called a semi-spheroidal shape anode). During 1-4 h of exposure at $800-1250{ }^{\circ} \mathrm{C}$ preliminary annealing of the body is carried out which is followed by shrinkage and pore formation, and integrated material structure is formed. Finally, the body is sintered at 1350 $1450{ }^{\circ} \mathrm{C}$ in air or inert gas during $2-5 \mathrm{~h}$.

Stress and strain distributions in an anode of the proposed semi-spheroidal shape (Fig. 1) were calculated by finite element analysis (FEA) using software for calculating three-dimensional tasks Mechanical Desktop 6 Power Pack. Initial conditions were chosen similarly to previously substantiated for a spheroidal shape anode [23] as follows.

Material of $\mathrm{ZrO}_{2}-\mathrm{Y}_{2} \mathrm{O}_{3}-\mathrm{Ni}$ system; average value of ultimate fracture stress $\sigma_{f}=110 \mathrm{MPa}$; Young's modulus $E=1.5 \cdot 10^{5} \mathrm{MPa}$; Poisson's coefficient $v=0.3$; fixing pressure $f=1 \mathrm{MPa}$ along the closed-loop fixing of inner radius $R_{p}=10.5 \mathrm{~mm}$; external gas pressure $p=1 \mathrm{MPa}$ on the anode working surface; radius of the cylindrical part of anodes $R_{c}=12 \mathrm{~mm}$; spheroid (spherical cap) radii, $R$, were 36, 60, 180, 240, and $600 \mathrm{~mm}$, respectively.

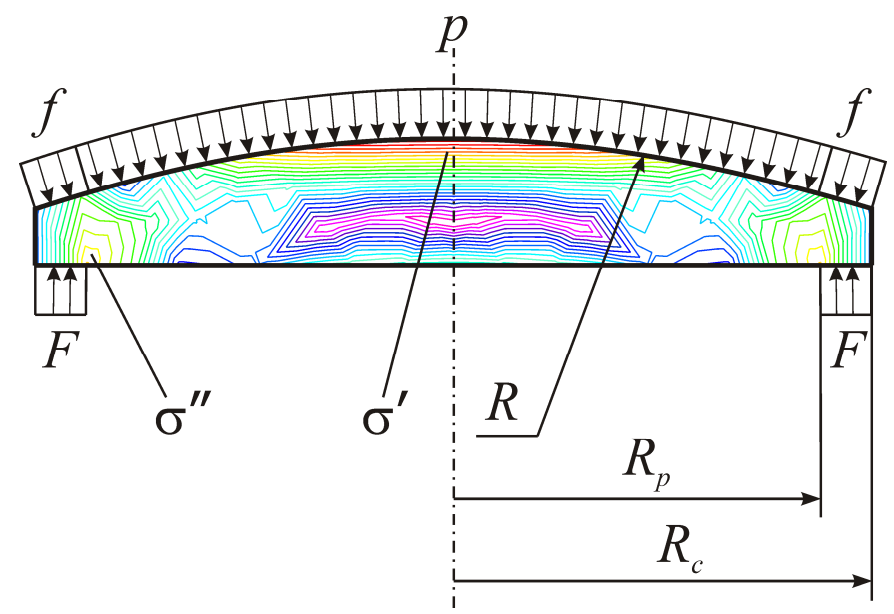

Fig. 1. Diagrams of application of fixing pressure, $f$, along the closed-loop fixing of inner radius, $R_{p}$, external gas pressure, $p$, on the anode working area, reaction force, $F$, along the closed-loop fixing, stress distribution along the axis $\sigma^{\prime}$ and the closed-loop fixing $\sigma^{\prime \prime}$ in the anode of a cylinder radius, $R_{c}$, with a flat bottom working surface and a convex top surface of a semi-spheroid radius, $R$. Stress distribution isolines obtained using FEA calculation are presented in the anode cross-section in colors

To determine stress and strain features of an anode of the semi-spheroidal shape and compare to those of the spheroidal shape anode [23], the following parameters were calculated:

- volume $V_{c}$ of an anode of the cylindrical shape:

$$
V_{c}=\pi \cdot R_{c}^{2} \cdot h
$$


where $h$ is a height of a cylinder;

- volume $V_{s}$ of a convex part (spherical cap) of an anode of semi-spheroidal shape:

$$
V_{s}=\frac{1}{3} \pi \cdot H^{2}(3 R-H),
$$

where $H$ is a hight of a convex part (spherical cap) of the anode;

- volume $V$ of an anode of semi-spheroidal shape:

$$
V=\pi \cdot R_{c}^{2} \cdot h+\frac{1}{3} \pi \cdot H^{2}(3 R-H) ;
$$

- volume $V^{\prime}$ of an anode of spheroidal shape:

$$
V^{\prime}=\pi \cdot R_{c}^{2} \cdot h+\frac{2}{3} \pi \cdot H^{2}(3 R-H) ;
$$

- ratio $S / S_{c}$ of the semi-spheroidal shape anode surface area to the surface area of an anode of the cylindrical shape:

$$
\frac{S}{S_{c}}=\frac{R \cdot H+0.5 \cdot R_{c}^{2}+R_{c} \cdot H}{R_{c}^{2}+R_{c} \cdot H} ;
$$

- ratio $S^{\prime} / S_{c}$ of the spheroidal shape anode surface area to the surface area of an anode of the cylindrical shape:

$$
\frac{S^{\prime}}{S_{c}}=\frac{2 \cdot R \cdot H+R_{c} \cdot H}{R_{c}^{2}+R_{c} \cdot H}
$$

- ratio $V / V_{c}\left(V^{\prime} / V_{c}\right)$ of the semi-spheroidal (or spheroidal) shape anode volume to the volume of an anode of the cylindrical shape;

- ratio $V / V_{c l}\left(V^{\prime} / V_{c l}\right)$ of the semi-spheroidal (or spheroidal) shape anode volume to the volume of an anode of the cylindrical shape, where $V_{c l}=452 \mathrm{~mm}^{3}$ is the calculated volume of the cylindrical anode of radius $R_{c}=12 \mathrm{~mm}$ and of height $1 \mathrm{~mm}$ fabricated for industrial applications [20].

\section{Results and Discussion}

Based on the calculated stress-strain and geometric parameters we obtained a graph of calculated values of $S / S_{c}$ ratio for the semi-spheroidal shape anodes (series A) at a variety of $R / R_{c}$ ratios (Fig. 2, curve 1). It can be seen that this ratio changes from 1 to 1.004 while decreasing $R / R_{c}$ ratio from 20 to 5 . Further decrease in $R / R_{c}$ ratio from 5 to 3 is followed by a noticeable increment of anode surface area and, respectively, the change in $S / S_{c}$ ratio (up to 1.013) although the increase in $S / S_{c}$ ratio is not as considerable as in $S^{\prime} / S_{c}$ ratio calculated for the spheroidal shape anodes (series B) where it reaches a value of 1.026 (Fig. 2, curve 2). However, the semi-spheroidal shape anodes take advantage over the spheroidal ones in terms of $V / V_{c l}$ ratio of the corresponding anode volume to the volume of the in-production cylindrical shape anode of a radius of $12 \mathrm{~mm}$ and $1 \mathrm{~mm}$ thickness. Its slight increase (from 1.15 to 1.61 ) with decreasing $R / R_{c}$ ratio from 20 to 5 (Fig. 2, curve 3) as compared to the more steep change for the spheroidal shape anodes ( $V^{\prime} / V_{c l}$ ratio from 1.30 to 2.21, curve 4) could serve as an important asset when improving functional characteristics of an anode, in particular, its electrochemical performance. In the same manner, we can compare anodes of both the shapes when $R / R_{c}$ ratio ranges from 5 to 3 . It can be seen that the spheroidal shape anodes have a significant limitation since a steep increase in anode volume occurs due to the excessive convexity $\left(V^{\prime} / V_{c l}\right.$ from 2.21 to 2.77 ) already in the $R / R_{c}$ range from 5 to 3.5 (hatched region 6 in Fig. 2), that does not provide the conditions for reactions to be passed in the fuel cell. In contrast to this, the semi-spheroidal shape anodes do not have such a strong limitation since in the $R / R_{c}$ range from 5 to 3 (hatched region 5 in Fig. 2), a gentler slope of the corresponding $V / V_{c l}$ dependence (Fig. 2, curve 4) occurs exhibiting the moderate convexity ( $V / V_{c l}$ is still about 2 ) at $R / R_{c}$ ratio of 3, that allows the reactions to be passed.

The volumes $V$ and $V_{c}$ and $V / V_{c}$ ratios were calculated as functions of parameters $R$ and $R_{c}$ $\left(R_{c}=12 \mathrm{~mm}\right)$ and variable anode height (its increment step was $0.5 \mathrm{~mm}$ ). For each partial case, the stress distribution and maximum strain were calculated by finite element analysis. Then the stresses in the most 
dangerous areas (i.e. along the axis $\sigma^{\prime}$ and the closed-loop fixing $\sigma^{\prime \prime}$, see Fig. 1) were determined. A parameter $\sigma / \sigma_{f}$ of the stress distribution called the relative strength was calculated as the ordinary $(\sigma)$ to fracture $\left(\sigma_{f}\right)$ stresses ratio. Three-dimensional (3D) dependences of values of $\sigma / \sigma_{f}$ ratio on the calculated values of $V / V_{c}$ ratio at a variety of $R / R_{c}$ ratios for the semi-spheroidal shape anodes (series A) were plotted (Fig. 3). Based on these curves, 3D surfaces of stress distribution $\sigma / \sigma_{f}$ along the axis (Fig. 3, surface 1) and closed-loop fixing (Fig. 3, surface 2) in semi-spheroidal shape anodes were constructed. As a result of the graphic intersections of these surfaces, three-dimensional intersection curves ( 3 and 4$)$ with their projections on the coordinate planes $\left[R / R_{c} ; V / V_{c}\right],\left[\sigma / \sigma_{f} ; R / R_{c}\right]$, and $\left[\sigma / \sigma_{f} ; V / V_{c}\right]$ (curves $3^{\prime}$ and 4', 3" and 4", and 3"' and 4'", respectively) were also plotted. The curves display the values of balanced stresses depending on $V / V_{c}$ and $R / R_{c}$ parameters. Domains of these curves were also defined.

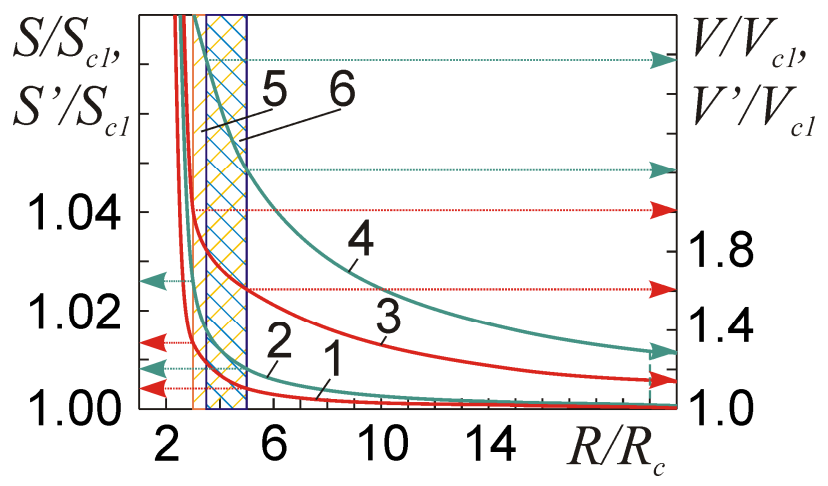

Fig. 2. A graph of calculated values of the ratios $S / S_{c}$ and $S^{\prime} / S_{c}$ for the semi-spheroidal and spheroidal shape anodes (curves 1 and 2, respectively; an ordinate axis is on the left) and the ratios $V / V_{c l}$ and $V^{\prime} / V_{c l}$ of the corresponding anode volume to the volume of the in-production cylindrical shape anode of a radius of $12 \mathrm{~mm}$ and of $1 \mathrm{~mm}$ thickness (curves 3 and 4, respectively; an ordinate axis is on the right) depending on the spheroid to cylinder radii ratio, $R / R_{c}$.

The hatched regions 5 and 6 correspond to the semi-spheroidal and spheroidal shape anodes, respectively

$3 \mathrm{D}$ isolines showing stress distribution along the axis (curve 5 on surface 1) and closed-loop fixing (curve 6 on surface 2) in anodes with a $1 \mathrm{~mm}$ thick cylindrical part at a variety of $R / R_{c}\left(V / V_{c}\right)$ ratios were plotted. It can be seen that these curves are intersected at $R / R_{c}$ ratio of about 5 . Thus, for this geometry of the anode, stress along the closed-loop fixing is lower than along the axis, at a low $R / R_{c}$ ratio, and becomes higher than that, when this ratio is higher than 5 .

Projections of the curves on the coordinate planes $\left[V / V_{c} ; R / R_{c}\right],\left[\sigma / \sigma_{f} ; R / R_{c}\right]$, and $\left[\sigma / \sigma_{f} ; V / V_{c}\right]$ were approximated by the corresponding lines (Fig. 3, curves 3' and 4', 3" and 4", and 3"' and 4"', respectively).

Curve 3 ' for the ranges $1.01<\left(V / V_{c}\right)<1.51$ and $5<\left(R / R_{c}\right)<27$ was described by an equation:

$$
V / V_{c}=1.9425 \cdot\left(R / R_{c}\right)^{-0.177} \text {. }
$$

Curve 4' for the ranges $1.0<\left(V / V_{c}\right)<1.4$ and $3<\left(R / R_{c}\right)<27$ was described by an equation:

$$
V / V_{c}=1.5094 \cdot\left(R / R_{c}\right)^{-0.119} \text {. }
$$

Curve 3" for the ranges $0.16<\left(\sigma / \sigma_{f}\right)<0.32$ and $5<\left(R / R_{c}\right)<50$ was described by an equation:

$$
\sigma / \sigma_{f}=0.0983 \cdot\left(R / R_{c}\right)^{0.3035} \text {. }
$$

Curve 4 " for the ranges $0.038<\left(\sigma / \sigma_{f}\right)<0.052$ and $3<\left(R / R_{c}\right)<50$ was described by an equation:

$$
\sigma / \sigma_{f}=0.0621 \cdot\left(R / R_{c}\right)^{-0.106} \text {. }
$$
equation:

Curve 3"' for the ranges $0.16<\left(\sigma / \sigma_{f}\right)<0.32$ and $1.01<\left(V / V_{c}\right)<1.51$ was described by an

$$
\sigma / \sigma_{f}=0.3015 \cdot\left(V / V_{c}\right)^{-1.618}
$$

Curve 4"' for the ranges $0.038<\left(\sigma / \sigma_{f}\right)<0.053$ and $1.0<\left(V / V_{c}\right)<1.4$ was described by an equation:

$$
\sigma / \sigma_{f}=0.0444 \cdot\left(V / V_{c}\right)^{0.6403}
$$




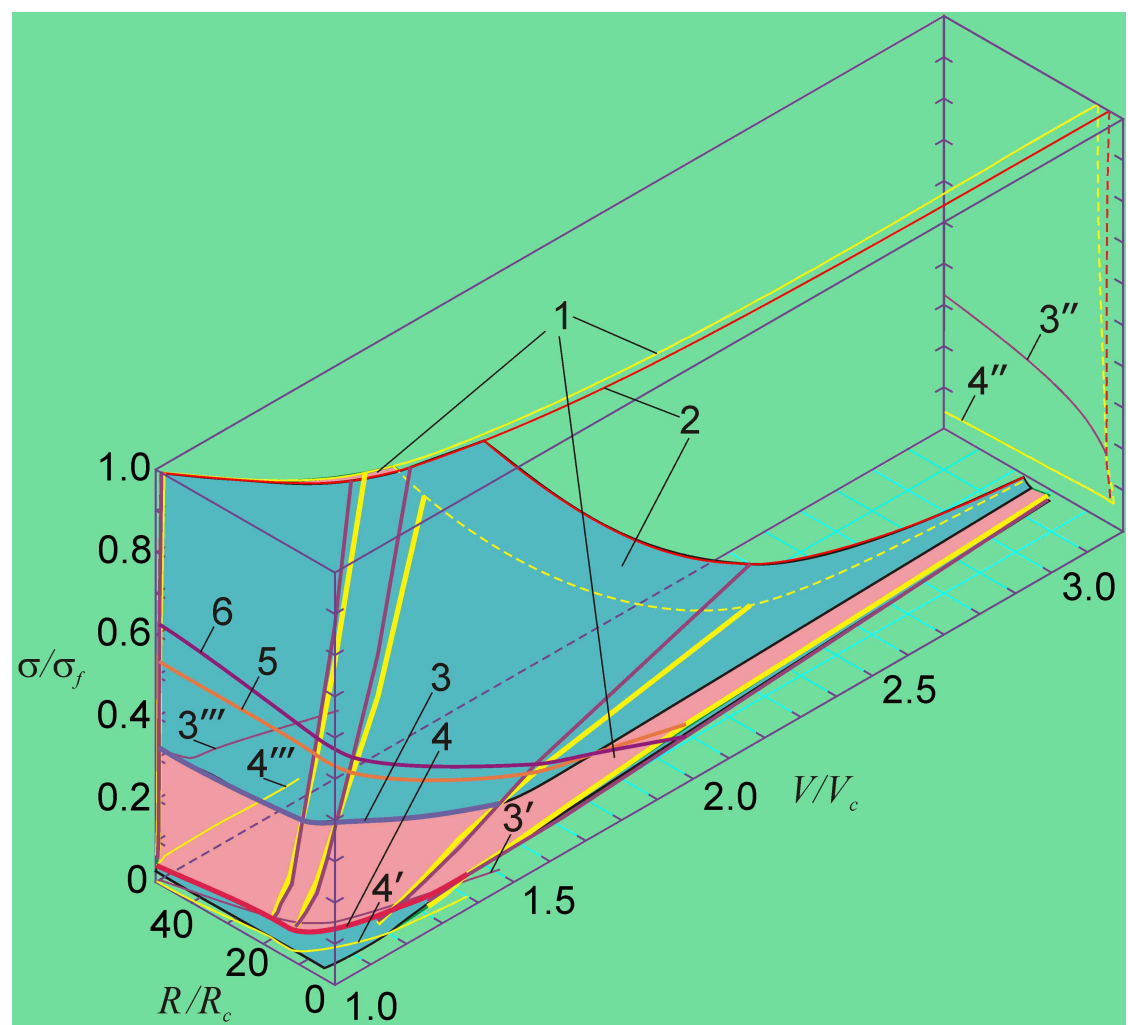

Fig. 3. Surfaces of stress distribution $\sigma / \sigma_{f}$ along the axis (surface 1) and closed-loop fixing of semi-spheroidal shape anodes (surface 2), as well as their three-dimensional intersection curves (3 and 4) with their projections on the coordinate planes $\left[R / R_{c} ; V / V_{c}\right],\left[\sigma / \sigma_{f} ; R / R_{c}\right]$, and $\left[\sigma / \sigma_{f} ; V / V_{c}\right]$ (curves 3' and 4', 3" and 4", and 3"' and 4"', respectively). Curves 5 and 6 (located on surfaces 1 and 2, respectively) show levels of stresses in anodes with a

$1 \mathrm{~mm}$ thick cylindrical part at a variety of $R / R_{c}\left(V / V_{c}\right)$ ratios

In the same manner, 3D dependences of values of maximum strain, $\varepsilon_{\max }$, on the calculated values of $V / V_{c}$ ratio at a variety of $R / R_{c}$ ratios for the semi-spheroidal shape anodes (series A) were plotted (Fig. 4). Based on these curves, a 3D surface of maximum strain along the axis in semi-spheroidal shape anodes was constructed (Fig. 4, surface 1). Also, a 3D surface of maximum strain in spheroidal shape anodes (series B) was constructed for comparison (Fig. 4, surface 2). No intersection of these two surfaces was found. It means that surface 1 is shifted graphically towards higher values of maximum strain at the same geometric parameters.

3D isolines showing strain levels in semi-spheroidal (series A) and spheroidal shape anodes (series B) with the $0.5 \mathrm{~mm}$ (curves 3 and 4 , respectively) and $1 \mathrm{~mm}$ thick cylindrical parts (curves 5 and 6 , respectively) and a variety of $R / R_{c}$ ratios, as well as multiple $3 \mathrm{D}$ isolines corresponding to other thicknesses (an increment step is $0.5 \mathrm{~mm}$ ) of semi-spheroidal shape anodes were plotted. The projections of curves 5 and 6 on the coordinate planes $\left[R / R_{c} ; V / V_{c}\right],\left[\sigma / \sigma_{f} ; R / R_{c}\right]$, and $\left[\sigma / \sigma_{f} ; V / V_{c}\right]$ (Fig. 4, curves $5^{\prime}$ and 6', 5" and 6", and 5"' and 6"', respectively) were also plotted. On an example of anodes of both the shapes with a $1 \mathrm{~mm}$ thick cylindrical part, curves 5 and 6 and all their projections show in detail a graphical shift of the constructed 3D surfaces relative to each other.

Based on 3D dependences of values of $\sigma / \sigma_{f}$ ratio for the semi-spheroidal shape anodes (series A) on the calculated values of $V / V_{c}$ ratio at a variety of $R / R_{c}$ ratios (Fig. 3) and the same dependences for the spheroidal shape anodes (series B) [23], simplified 2D dependences of values of $\sigma / \sigma_{f}$ ratio on the $V / V_{c}$ ratio for anodes of these shapes for both the cases of stress distribution along the axis and closed-loop fixing were plotted (Fig. 5, a). It is seen that at low $R / R_{c}$ ratio and, respectively, a higher $V / V_{c}$ ratio, levels of $\sigma / \sigma_{f}$ ratio are quite low (of about $0.4-0.12$ at $R / R_{c}=3$ ) for both the anode shapes whereas with increasing $R / R_{c}$ ratio, stresses increase more steeply in the semi-spheroidal shape anodes. Besides, almost for all the calculated geometries, the stresses along the axis become lower than along the closed-loop fixing, while increasing both $V / V_{c}$ and $R / R_{c}$ ratios. 


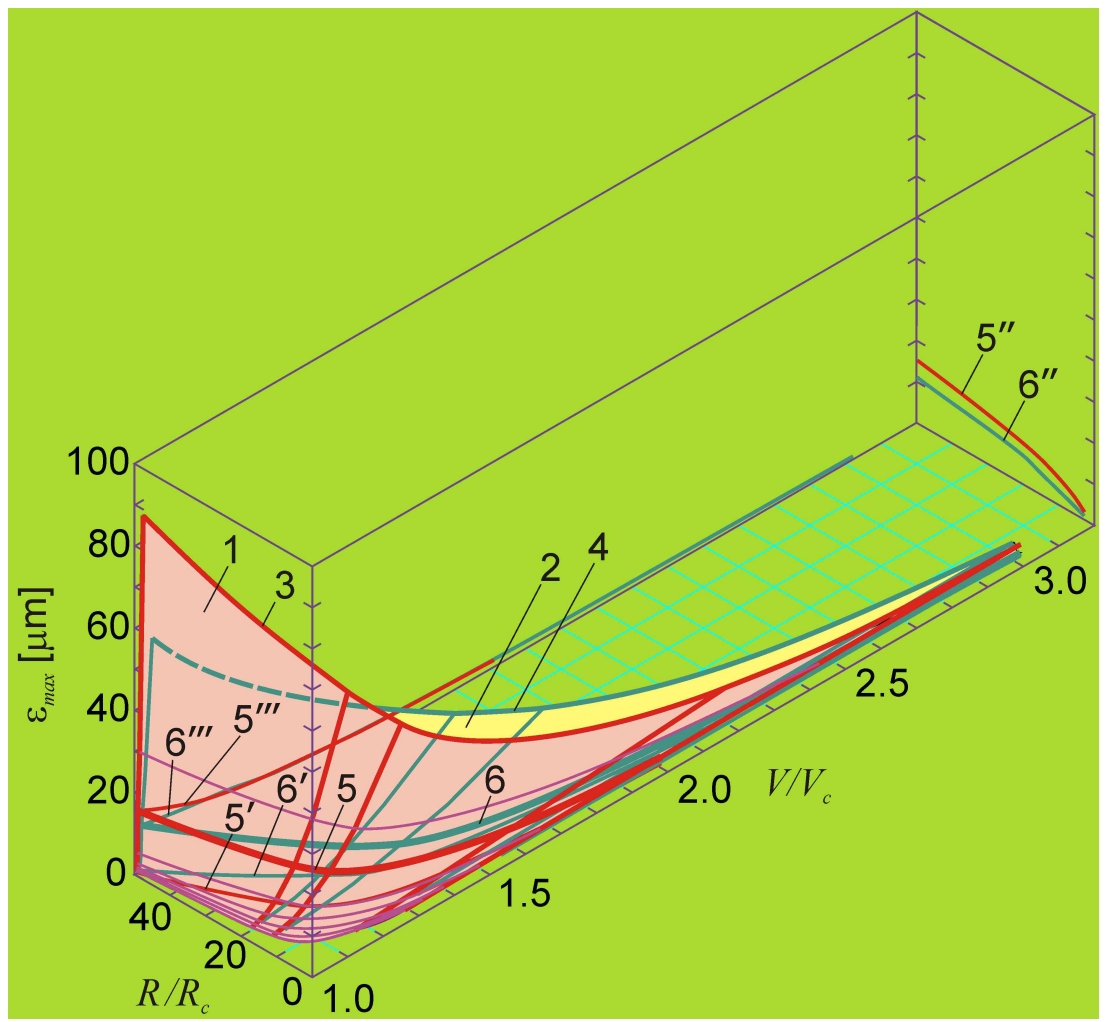

Fig. 4. Surfaces of the maximum strain, $\varepsilon_{\max }$, along the axis of semi-spheroidal shape anodes (surface 1) and spheroidal shape anodes (surface 2) [23], as well as their three-dimensional isolines showing levels of the strain in anodes with the $0.5 \mathrm{~mm}$ (curves 3 and 4, respectively) and $1 \mathrm{~mm}$ thick cylindrical parts (curves 5 and 6 , respectively) at a variety of $R / R_{c}\left(V / V_{c}\right)$ ratios, with their projections on the coordinate planes $\left[R / R_{c} ; V / V_{c}\right],\left[\sigma / \sigma_{f} ; R / R_{c}\right]$, and $\left[\sigma / \sigma_{f} ; V / V_{c}\right]$ (curves 5' and 6', 5" and 6", and 5"' and 6"', respectively)

Also, 2D dependences of values of $\sigma / \sigma_{f}$ ratio on the $V / V_{c}$ ratio (isolines) for anodes of series A and B with the $0.5 \mathrm{~mm}, 1 \mathrm{~mm}$, and $1.5 \mathrm{~mm}$ thick cylindrical parts at a variety of $R / R_{c}$ ratios for both the cases of stress distribution along the axis (Aa and Ba series, Fig. 5, b) and closed-loop fixing (As and Bs series, Fig. 5, b) were plotted. The graph (Fig. 5, b) shows that there exists an area of geometric parameters that allows the appropriate stress level to be reached ensuing safe long-term operation of the semi-spheroidal shape anode. The domain of this area was graphically defined: for $V / V_{c}$ ratio in the range from 1.2 to 2.2, $R / R_{c}$ ratio is in the range from 0.5 to 1.5 , whereas $\sigma / \sigma_{f}$ ratio is below 0.25 .

In the same way, 2D dependences of the maximum strain, $\varepsilon_{\max }$, along the axis of semi-spheroidal shape anodes (series A) and spheroidal shape anodes (series B) [23] on the $V / V_{c}$ ratio, at a variety of $R / R_{c}$ ratios, were plotted (Fig. 6). Based on the constructed isolines showing levels of strain in anodes with the $0.5 \mathrm{~mm}, 1 \mathrm{~mm}$, and $1.5 \mathrm{~mm}$ thick cylindrical parts at a variety of spheroid to cylinder radii ratios, it can be concluded that a semi-spheroidal shape anode has an advantage over spheroidal one, since the last is inferior to it in term of relative volume increase at the same level of maximum strain. This feature allows ensuing the better electrochemical behavior of a SOFC.

If accidentally, a crack is initiated at the edge of an anode, it may advance depending on the crack profile geometry and loading conditions. It was found by us earlier using a shape-dependent crack deceleration approach [23], [24], that the spheroid shaped SOFC anode-substrate has a higher resistance to crack advance as compared to a conventional flat one. Based on some resemblance in stress distribution patterns found for both the semi-spheroidal and spheroidal shape anodes it can be concluded that, somewhat like the spheroidal one, the semi-spheroidal shape anode also has a higher resistance to advancing crack as compared to a flat one. A crack is already decelerated if the current value of the crack profile angle is about 10 degrees [24], similar to the case of spheroidal shape anode.

Thus, a semi-spheroidal shape anode allows the stress distribution to be changed and a level of maximum strain in a fuel cell to be lowered. Therefore, the deformation resistance of a fuel cell and its 
lifetime increase. In such a way, the cost of electricity that the fuel cell produces may be reduced and its efficiency may be increased, as compared to that of a fuel cell comprising an anode-substrate of the cylindrical shape.

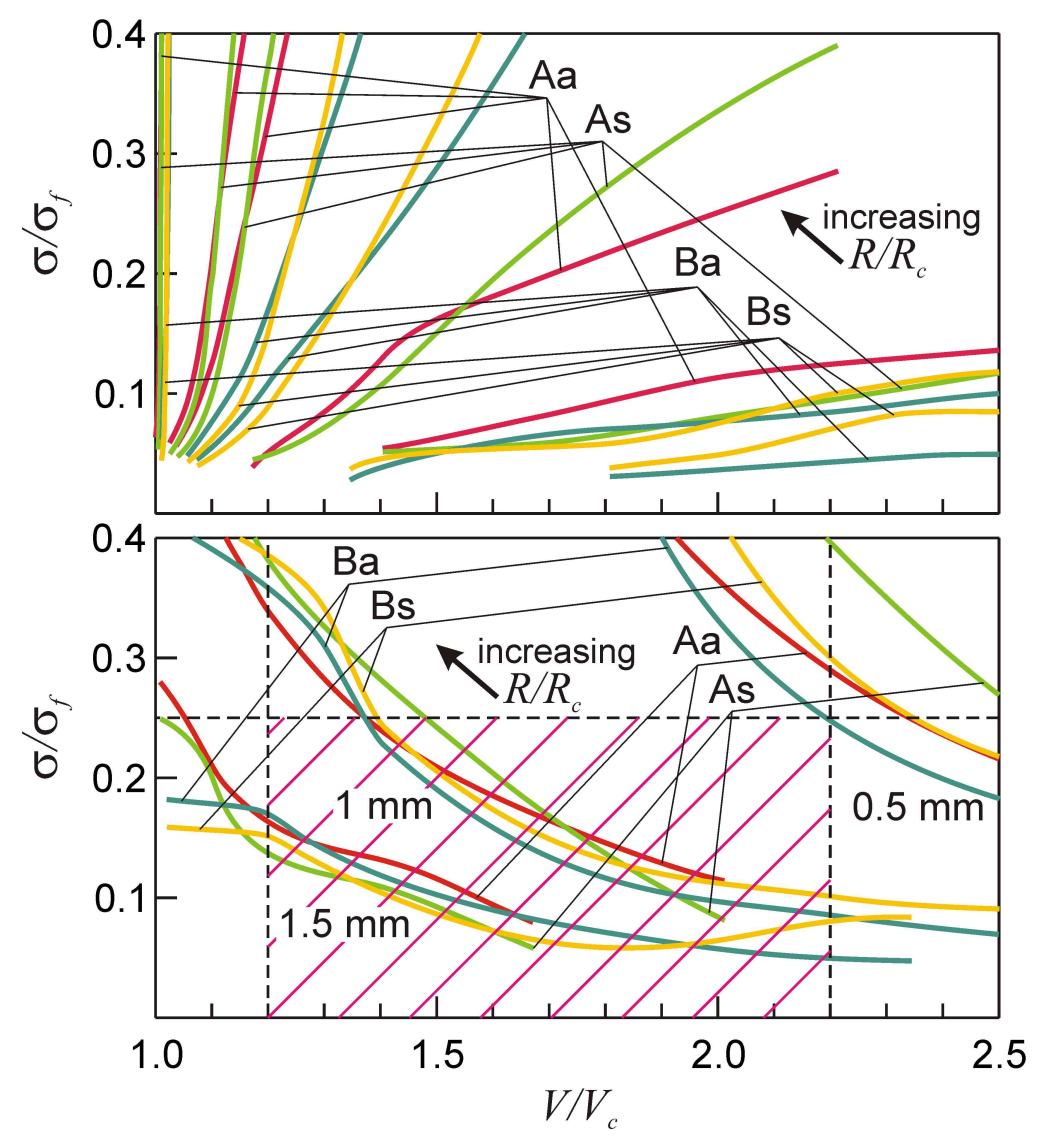

Fig. 5. Dependences of (a) stress distribution along the axis (Aa, Ba) and closed-loop fixing (As, Bs) of semispheroidal shape anodes (series A) and spheroidal shape anodes (series B) [23] on the $V / V_{c}$ ratio, as well as (b) the isolines showing levels of stress distribution along the axis (Aa, Ba) and closed-loop fixing (As, Bs) in anodes of series A and B with the $0.5 \mathrm{~mm}, 1 \mathrm{~mm}$, and $1.5 \mathrm{~mm}$ thick cylindrical parts at a variety of $R / R_{c}$ ratios. A hatched area (b) denotes the appropriate stress level $\sigma / \sigma_{f}$ and corresponding ranges of $V / V_{c}\left(R / R_{c}\right)$ ratios proposed for designing anodes of both the shapes (series $\mathrm{A}$ and $\mathrm{B}$ )

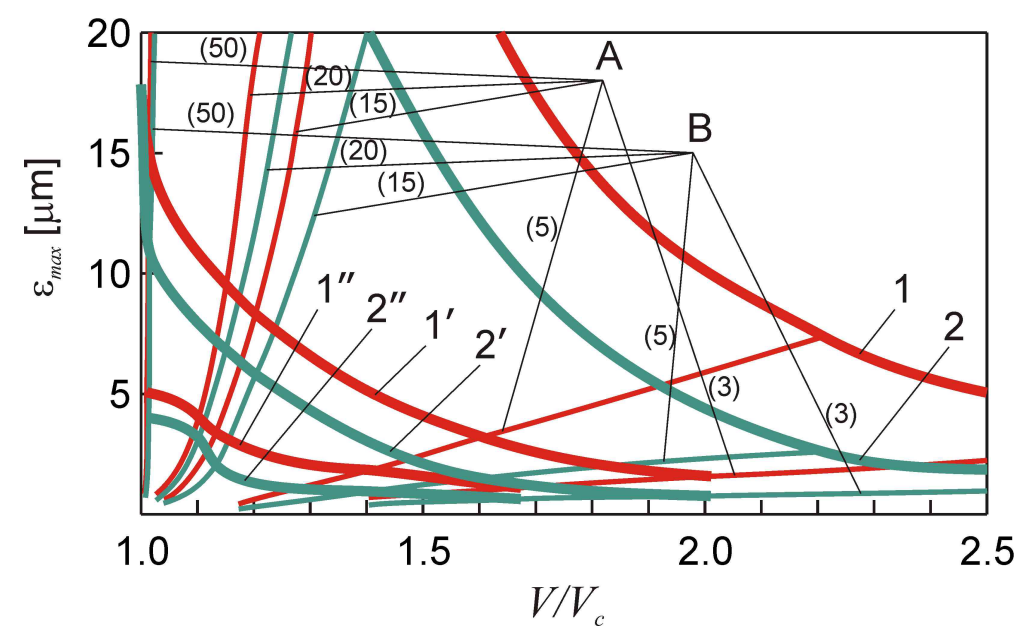

Fig. 6. Dependences of the maximum strain, $\varepsilon_{\max }$, along the axis of semi-spheroidal shape anodes (series A) and spheroidal shape anodes (series B) [23] on the $V / V_{c}$ ratio, as well as the isolines showing levels of the strain in anodes with the $0.5 \mathrm{~mm}$ (curves 1 and 2, respectively), $1 \mathrm{~mm}$ (curves 1 ' and 2', respectively), and $1.5 \mathrm{~mm}$ thick cylindrical parts (curves 1'" and 2', respectively) at a variety of $R / R_{c}$ ratios. The numbers in brackets indicate $R / R_{c}$ magnitudes for corresponding curves 


\section{Conclusions}

In this work, stress and strain distributions in a solid oxide fuel cell anode of a semi-spheroidal shape loaded with a fixing pressure along the closed-loop fixing and with an external gas pressure applied to the anode working surface were calculated by finite element analysis. Based on these data, three-dimensional (3D) surfaces of stress distribution along the axis and closed-loop fixing of semi-spheroidal shape anodes were constructed. It is found that there exists an area of geometric parameters that allows the appropriate stress level to be reached ensuing safe long-term operation of the semi-spheroidal shape anode. The domain of this area is as follows: for a semi-spheroid to cylinder volumes ratio $V / V_{c}$ in the range from 1.2 to 2.2 , a spheroid to cylinder radii ratio $R / R_{c}$ is in the range from 0.5 to 1.5 , whereas the ordinary to fracture stresses ratio $\sigma / \sigma_{f}$ is below 0.25 .

The constructed isolines of strain levels in anodes with the $0.5 \mathrm{~mm}, 1 \mathrm{~mm}$, and $1.5 \mathrm{~mm}$ thick cylindrical parts at a variety of spheroid to cylinder radii ratios $R / R_{c}$ showed an advantage of a semispheroidal shape anode over spheroidal one since the last is inferior to the semi-spheroidal one in term of relative volume increase $V / V_{c}$ at the same level of maximum strain. This feature allows ensuing the better electrochemical behavior of a SOFC.

As is the case with crack behavior in a spheroidal shape anode, a semi-spheroidal shape anode also has a higher resistance to advancing a crack as compared to a flat anode. A nucleated crack in a semispheroidal shape anode is already decelerated if the current value of the crack profile angle is about 10 degrees.

\section{References}

[1] R. Knibbe, et al., "Durability of solid oxide cells", Green, vol. 52, issue 2, pp. 5747-5756, Jan. 2011.

[2] A. Faes, et al., "A review of RedOx cycling of solid oxide fuel cells anode", Membranes, vol. 2, issue 3, pp. 585-664, Aug. 2012.

[3] Ye. Brodnikovskyi, et al., "Influence of reduction conditions of $\mathrm{NiO}$ on its mechanical and electrical properties", Journal of Electrochemical Science and Engineering, vol. 6, issue 1, pp. 113-121, Jan. 2016.

[4] Y. Wang, et al., "Effects of powder sizes and reduction parameters on the strength of Ni-YSZ anodes", Solid State Ionics, vol. 177, issues 17-18, pp. 1517-1527, Oct. 2006.

[5] M. Ettler, et al., "Durability of Ni anodes during reoxidation cycles", Journal of Power Sources, vol. 195, issue 17, pp. 5452-5467, Sept. 2010.

[6] A. Atkinson, et al., "Advanced anodes for high-temperature fuel cells", Nature Materials, vol. 3, issue 1, pp. 17-27, Jun. 2004.

[7] B. D. Vasyliv, "Improvement of the electrical conductivity of the material of anode in a fuel cell by the cyclic redox thermal treatment", Mater. Sci., vol. 46, issue 2, pp. 260-264, Nov. 2010.

[8] V. Ya. Podhurs'ka, et al., "Structural transformations in the NiO-containing anode of ceramic fuel cells in the course of its reduction and oxidation", Mater. Sci., vol. 49, issue 6, pp. 805-811, May 2014.

[9] V. Podhurska, B. Vasyliv, "Influence of $\mathrm{NiO}$ reduction on microstructure and properties of porous $\mathrm{Ni}-$ $\mathrm{ZrO}_{2}$ substrate", in Proc. Int. Conf. Oxide Materials for Electronic Engineering (OMEE-2012), Lviv, Ukraine, September 3-7, 2012, pp. 293-294.

[10] B. Vasyliv, et al., "Preconditioning of the YSZ-NiO fuel cell anode in hydrogenous atmospheres containing water vapor", Nanoscale Research Letters, vol. 12, issue 1, article number 265, Apr. 2017.

[11] A. Wood, D. Waldbillig, "Preconditioning treatment to enhance redox tolerance of solid oxide fuel cells", U.S. Patent 8029946 B2, October 04, 2011.

[12] B. D. Vasyliv, et al., "Sposib obrobky NiO-vmisnykh anodiv tverdooksydnoi palyvnoi komirky" ["A method of treatment of NiO-containing anodes for a solid oxide fuel cell"], UA Patent 78992, April 10, 2013. [in Ukrainian].

[13] J. Huiming, S. Maosong, "Study of integrity of $\mathrm{NiO}$ oxide film by acoustic emission method", in Proc. 5th Int. Conf. Natural Computation (ICNC-2009), Tianjin, China, August 14-16, 2009, vol. 6, pp. 252-256.

[14] V. Lawlor, et al., "Review of the micro-tubular solid oxide fuel cell: Part I. Stack design issues and research activities", Journal of Power Sources, vol. 193, issue 2, pp. 387-399, March 2009.

[15] R.-H. Song, D.-R. Shin, J.-H. Kim, "Anode-supported flat-tubular solid oxide fuel cell stack and fabrication method of the same”, U.S. Patent 7285347 B2, October 23, 2007. 
[16] Y. Bai, et al., "Dip coating technique in fabrication of cone-shaped anode-supported solid oxide fuel cells", Journal of Alloys and Compounds, vol. 480, issue 2, pp. 554-557, Sept. 2009.

[17] Y. Zhang, et al., "Redox cycling of Ni-YSZ anode investigated by TRP technique", Solid State Ionics, vol. 176, issues 29-30, pp. 2193-2199, Nov. 2005.

[18] M. Radovic, E. Lara-Curzio, "Mechanical properties of tape cast nickel-based anode materials for solid oxide fuel cells before and after reduction in hydrogen", Acta Materialia, vol. 52, issue 20, pp. 5747-5756, Jul. 2004.

[19] T. Miyazawa, "Flat-plate solid oxide fuel cell", U.S. Patent 20110091785 A1, April 21, 2011.

[20] B. D. Vasyliv, "A procedure for the investigation of mechanical and physical properties of ceramics under the conditions of biaxial bending of a disk specimen according to the ring-ring scheme", Mater. Sci., vol. 45, issue 4, pp. 571-575, Jul. 2009.

[21] B. Sun, et al., "Effect of thermal cycling on residual stress and curvature of anode-supported SOFCs", Fuel Cells, vol. 6, pp. 805-813, Jul. 2009.

[22] O. P. Ostash, B. D. Vasyliv, V. Ya. Podhurska, "Sposib vyhotovlennia anoda-pidkladky dlia palyvnoi komirky" ["A method of fabrication of an anode substrate for a fuel cell”], UA Patent 109256, August 25, 2016. [in Ukrainian].

[23] I. Kuzio, et al., "Substantiation of the shape of a solid oxide fuel cell anode using the stress-strain and shape-dependent crack deceleration approaches", Ukrainian Journal of Mechanical Engineering and Materials Science, vol. 5, no. 1, pp. 29-38, 2019.

[24] B. Vasyliv, Crack initiation and retardation in ceramics. Techniques and applications. Riga, Latvia: LAMBERT Academic Publishing, 2019. 